\title{
Corrigendum Skitmore M and Pemberton J (1994). A multivariate approach to construction contract bidding mark-up strategies
}

This paper contains the following errors, for which we apologise. We are grateful to Dr Shu-Lin Liu, Chinese Academy of Sciences, Beijing, for pointing this out to us.

1. Eqn (21) should read

$$
\hat{\beta}_{j}=\frac{\sum_{i=1}^{r} \delta_{i j}\left(y_{i j}-\alpha_{i}\right) / \sigma_{i}^{2}}{\sum_{i} \delta_{i j} / \sigma_{i}^{2}}
$$

In practice, however, convergence problems occur with this formulation. The original eqn (21) was used previously by one of us as an approximation.

2. Eqn (23) should read

$$
\hat{\sigma}_{i}^{2}=\frac{1}{n_{i}} \sum_{j} \delta_{i j}\left(y_{i j}-\alpha_{i}-\beta_{j}\right)^{2}
$$

3. Figure 1 legend should read:

$$
\begin{aligned}
& c_{e}(x) \\
& f_{55}(x) \\
& X_{e}=X_{e} v
\end{aligned}
$$

4. Figures 2 and $3 f_{s s}(x)$ should read $f_{55}(x)$.

University of Wales, Swansea

J Pemberton, Queensland University of Technology, M Skitmore.

\section{References}

1 Skitmore M and Pemberton J (1994). A multivariate approach to construction bidding mark-up strategies. J Opl Res Soc 45: $1263-1272$. 